\title{
ANÁLISIS DE LA AFECCIÓN DEL DEPORTE DE ESCALADA EN LA BIOLOGÍA REPRODUCTORA DEL QUEBRANTAHUESOS (GYPAETUS BARBATUS L.) EN EL PIRINEO CENTRAL (ARAGÓN-ESPAÑA)
}

\author{
I. Blasco Ibáñez y L.A. Longares Aladrén \\ Dpto de Geografía y Ordenación del Territorio \\ C/ Pedro Cerbuna 12. Universidad de Zaragoza. 50009 Zaragoza \\ lalongar@unizar.es \\ J.A. Gil Gallús \\ Fundación para la Conservación del Quebrantahuesos \\ Plza S. Pedro Nolasco 1, 4 F. 50001 Zaragoza \\ fcq@quebrantahuesos.org
}

\begin{abstract}
Resumen: En todas las poblaciones naturales de aves rapaces existen unidades reproductoras (UR) cuya productividad, entendida como pollos volados/año, es baja o nula. Las molestias humanas en las zonas de nidificación han sido una de las causas más relacionadas con el descenso de la productividad de muchas aves rapaces (Richardson y Miller, 1997). Según Arroyo y Razin (2006) las actividades humanas cercanas a los nidos del Quebrantahuesos (Gypaetus barbatus L.) pueden causar molestias en la reproducción. Los vigentes planes de recuperación de la especie en la Cordillera pirenaica contemplan acciones específicas para evitar el fracaso reproductivo, mediante la aplicación de medidas preventivas de factores de perturbación: molestias por batidas de caza, escalada deportiva, etc. Durante los últimos 10 años el deporte de escalada en Aragón (en todas sus modalidades) ha experimentado un aumento en cuanto al número de escaladores y sectores donde se práctica. Con el fin de conocer las posibles repercusiones de la escalada en roca en la conservación del Quebrantahuesos se ha recopilado información sobre sectores de escalada en la provincia de Huesca. Posteriormente dicha información ha sido geo-
\end{abstract}

Recibido: 13-12-13 Aceptado: 30-5-14. 
rreferenciada espacialmente y se le han aplicado diversos métodos de análisis geoespacial con técnicas SIG en el entorno ArcGis. El objetivo ha sido realizar una aproximación al análisis de la posible relación entre el deporte de escalada y la nidificación de la especie. De un total de 315 nidos conocidos de Quebrantahuesos en Aragón (2011), se ha comprobado que el $11,75 \%$ de estos $(n=37)$ podrían estar afectados por la escalada en roca.

Palabras clave: quebrantahuesos, sectores de escalada, productividad, pendiente, nidos, unidad reproductora, SIG.

\begin{abstract}
Within all bird of prey natural populations exist reproductive units (UR), whose productivity, understood as juveniles flown a year, is low or none. The effects of human disturbance in nesting areas has been one of the mostly related reasons with the decrease of productivity of many birds of prey (Richarson y Miller, 1997). According to Arroyo y Razin (2006), human activity nearby Bearded vulture nests (Gypaetus barbatus L.) can negatively affect reproduction. Current recovery plans for this spice in place in the Pyrenees range, include specific measures in order to avoid reproductive failure, by mean of the application of preventing measures for disturbing factors: hunting, sport climbing. During the last 10 years climbing in Aragon (across all varieties), has experienced an increase in number of people and sectors where it's practiced. With the aim of knowing potential impacts of rock climbing in the conservancy of Bearded vulture information regarding climbing zones in the province of Huesca has been compiled. Afterwards, such information has been georeferrenced and different geospatial methodologies with GIS techniques in the ArcGis environment had been applied. The objective has been taking a close approach of the analysis of the posible correlation effect between climbing and nesting of this specie. Out of a total of 315 Bearded vulture nest in Aragon (2011), it has been proven that $11,75 \%(n=37)$ could had been affected by climbing sports.
\end{abstract}

Keywords: bearded vulture, climbing sectors, productivity, slope, nest, reproductive unit, SIG.

\title{
1. Introducción y objetivos
}

Única ave osteófaga del planeta, el Quebrantahuesos (Gypaetus barbatus L.) es un buitre territorial que está presente en algunas regiones montañosas de Europa, Asia y África (Del Hoyo et al., 1994). Durante los últimos siglos las poblaciones del Paleártico occidental han sufrido un drástico proceso de regresión, que ha conducido a su declive y extinción en la mayoría de las áreas donde estaba presente (Antor et al., 2004). 
En Europa occidental posee cuatro poblaciones aisladas entre sí: tres autóctonas (Creta, Córcega y Pirineos) y una reintroducida en los Alpes (Gil et al., 2009). En España ocupaba en el pasado los principales macizos montañosos de la Península Ibérica: Cordillera Cantábrica, Sistema Ibérico, Sistema Central, Sierra Morena y Sistemas Bético y Penibético (Hiraldo et al., 1979, Sánchez-Castilla et al., 2008, Longares, 2011).

En la actualidad tan solo posee una población reproductora en los Pirineos (Navarra, Aragón y Cataluña). Esto provocó su catalogación como especie en Peligro de Extinción (Decreto 181/2005 y D. 139/2011) y la puesta en marcha de diferentes planes de conservación en España, uno de ellos el Plan de Recuperación del Quebrantahuesos en Aragón (D. 45/2003). Esta región posee la mitad de la población reproductora de Quebrantahuesos del Pirineo (España, Francia y Andorra) 74 unidades reproductoras, que dan una densidad de 6,5 unidades reproductoras $/ 1000 \mathrm{Km}^{2}$ para el territorio aragonés ocupado por la especie (datos de 2011).

Son varias las causas que limitan el crecimiento y la supervivencia de la especie: de forma indirecta y directa factores climáticos extremos durante la reproducción (Longares, 2011), muerte por intoxicaciones (Gil y De Frutos, 2008), muerte por colisión o electrocución por tendidos eléctricos (Gil, 2009), muerte por disparo de armas de fuego (Margalida et al., 2008), transformación del paisaje y desaparición de los usos tradicionales (Ostermman, 1998), reducción de la disponibilidad alimento, proximidad a núcleos de población (Longares, 2011) y molestias humanas en las zonas de nidificación (Gil, 2011). Estas últimas han sido una de las causas más relacionadas con el descenso de la productividad (entendida como pollos volados/año) de muchas aves rapaces (Richardson y Mi1ler, 1997). Según Arroyo y Razin (2006) las actividades humanas cercanas a los nidos del Quebrantahuesos (Gypaetus barbatus) pueden causar molestias en la reproducción.

En este sentido, se ha venido observando en los últimos 10 años un incremento de la actividad de escalada en Aragón, relacionado tanto con el aumento del número de escaladores federados como de los sectores donde ésta se práctica y el número de vías de escalada nuevas que han ido apareciendo. Ante esta situación, se planteó analizar la situación de las UR de Quebrantahuesos monitorizadas dentro del Plan de Recuperación del Quebrantahuesos en Aragón (BOA no 29 de 12 de Marzo de 2003) en el Prepirineo y Pirineo Aragonés (Figura 1), en relación con la actividad de escalada en Aragón, teniendo como objetivo final, valorar y zonificar el posible riesgo de afección que esta actividad deportiva ejerce en relación con la biología reproductora de la especie. 


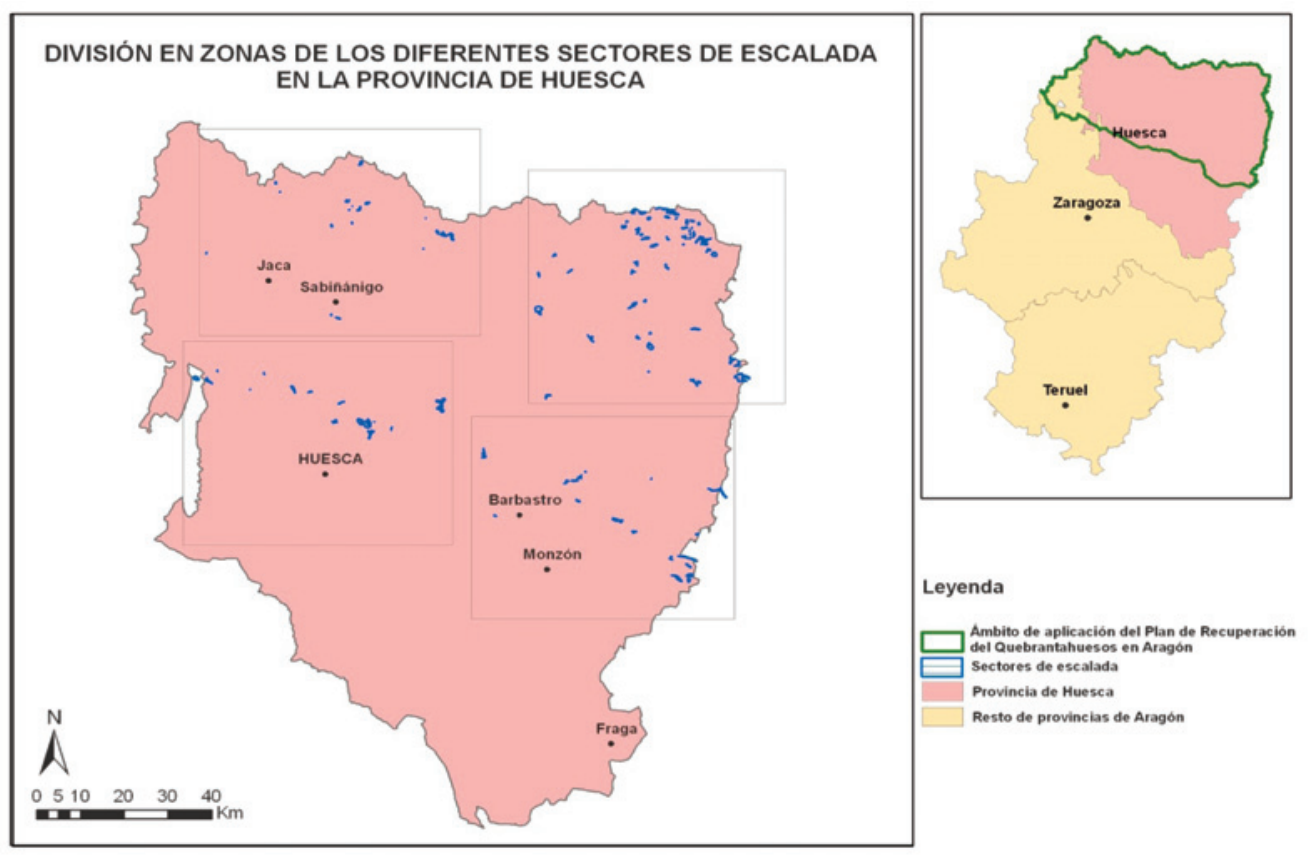

Figura 1. Mapa de localización del área de estudio y sectores de escalada diferenciados.

Fuente: Elaboración propia.

\section{Material y métodos}

Como se muestra en el esquema (Figura 2), durante las primeras fases del estudio se procedió a la recopilación de la información disponible sobre sectores de escalada en diferentes fuentes, así como sobre la ubicación y datos de reproducción de las UR de Quebrantahuesos en la provincia de Huesca, disponible en la Base de Datos del Quebrantahuesos actualizada y gestionada por la Fundación para la Conservación del Quebrantahuesos (FCQ) y el Gobierno de Aragón (GA).

La ausencia de un registro oficial de los lugares de escalada tanto en Aragón como a escala nacional, obligó a realizar una búsqueda indirecta de la información relacionada con los lugares de escalada. En un primer momento se contactó con el servicio de Biodiversidad del Gobierno de Aragón y con la FAM (Federación Aragonesa de Montañismo), donde se confirmó la ausencia de información y de un registro detallado de lugares donde se realiza la escalada. A partir de ese momento se inició una búsqueda exhaustiva en la bibliografía existente en el mercado y bibliotecas especializadas, así como a través de los diferentes blogs de escalada existentes en la red y que se utilizan con mucha frecuencia para comentar y difundir los enclaves y características de las vías de escalada. 


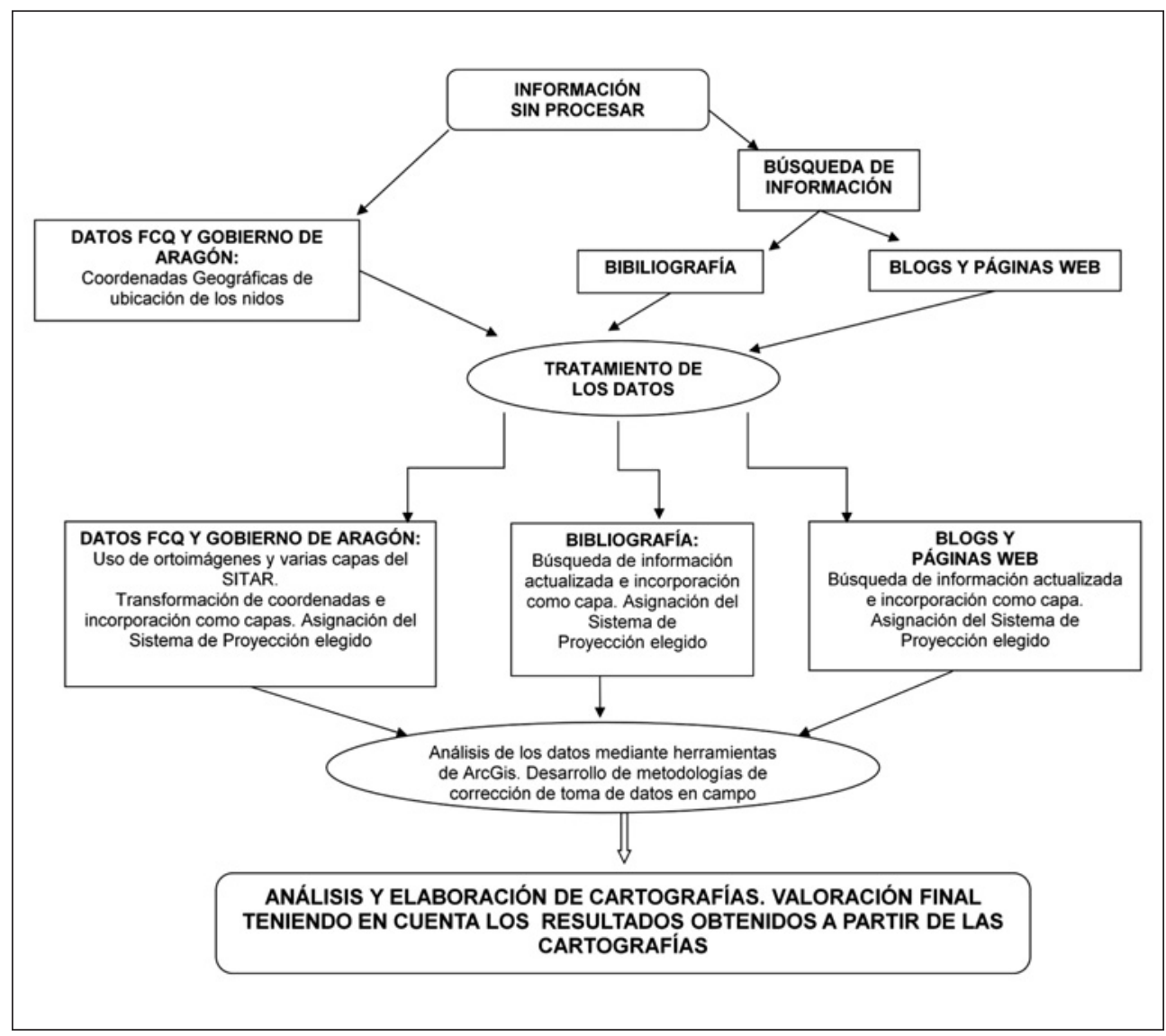

Figura 2. Esquema metodológico

Fuente: Elaboración propia.

La búsqueda de la información sobre escalada, se diseño para que al mismo tiempo sirviera como filtro para la obtención de la información disponible, lo más actualizada posible, para lo cual se seleccionó como criterio de partida la obra de Guinda y Logroño (2012), en la que se plantea una zonificación por sectores de escala del territorio analizado, adoptando de esta manera la delimitación espacial para abordar el presente trabajo. Esta información de partida, se completó a través de la consulta de otros textos y documentación que figuran en la Tabla 1. 
Tabla 1. Sectores de escalada definidos y webs con información sobre los mismos

\begin{tabular}{|c|c|}
\hline Sector de escalada & Web \\
\hline Alins (Las Paules) & http://luichy-lanochedelloro2.blogspot.com.es \\
\hline Alquézar & http://www.yumping.com/escalada/huesca/alquezar \\
\hline Ampriu & http://rumbopatagonia.blogspot.com.es \\
\hline Arguis & http://aragonenvertical.blogspot.com.es \\
\hline Balaitus & http://misterroresfavoritos.blogspot.com.es \\
\hline \multirow[t]{2}{*}{ Baldellou } & http://escaladatradicional.blogspot.com.es \\
\hline & http://www.baldellou.es \\
\hline Bentue de Rasal & http://aragonenvertical.blogspot.com.es \\
\hline Bielsa, La Foz de la Canal & http://www.climbingaway.fr \\
\hline Bujaruelo & http://www.enlavertical.com/escuelas \\
\hline Benabarre, El Molino & http://siempreparriba.blogspot.com.es \\
\hline Canelles (Estopiñan) & http://www.capitancabernicola.blogspot.com.es \\
\hline Castillonroi & http://luichy-lanochedelloro2.blogspot.com.es \\
\hline Cienfuens & http://www.escalismo.com \\
\hline Coll de Ladrones & http://escaladaparatodos.blogspot.com.es \\
\hline Las Devotas & http://www.caranorte.com \\
\hline Embún & http://croquisvallearagon.blogspot.com.es \\
\hline Escales & http://lanochedelloro.com \\
\hline Escarrilla & http://www.mundoglaciar.es \\
\hline Estadilla & http://carrodillasostenible.wordpress.com \\
\hline Forronías & http://www.enlavertical.com \\
\hline Hoz de Jaca & http://passe-murailles.org \\
\hline Llauset & http://lanochedelloro.com \\
\hline Liguerre de Cinca & http://raulsakisworld.blogspot.com.es \\
\hline Merli (La Puebla de Roda) & http://luichy-lanochedelloro2.blogspot.com.es \\
\hline Montrebei (Pared de Aragón) & http://www.mendiak.net \\
\hline Ordesa Fraucata & http://korkuerika.blogspot.com.es \\
\hline Panticosa & http://mirracom.blogspot.com.es \\
\hline \multirow[t]{3}{*}{ Predicadera } & http://trini-salva.blogspot.com.es \\
\hline & http://www.guara.info \\
\hline & http://vladimirbustof.blogspot.com.es \\
\hline Rapún & http://usuarios.arsystel.com \\
\hline Riglos & http://www.todoescalada.net \\
\hline Salto del Roldán & http://guaravertical.blogspot.com.es \\
\hline
\end{tabular}




\begin{tabular}{|l|l|}
\hline Sector de escalada & Web \\
\hline Santa Ana & $\mathrm{http}: / /$ www.canyoning-club.com \\
Sierra de Sis (Cajigar) & $\mathrm{http}: / /$ luichy-lanochedelloro2.blogspot.com.es \\
Sopeira & $\mathrm{http}: / /$ excarviclasica.blogspot.com.es \\
Telera & $\mathrm{http}: / /$ www.escalismo.com \\
& $\mathrm{http}: / /$ anayetvertical.com \\
Tramacastilla de Tena y & $\mathrm{http}: / /$ www.grifone.eu \\
Peña Bubón & $\mathrm{http}: / /$ www.mundoglaciar.es \\
Vadiello & $\mathrm{http}: / /$ nuestrolibrodepiadas.blogspot.com.es \\
Valcheladas & $\mathrm{http}: / /$ guaravertical.blogspot.com.es \\
Valle de Isábena & $\mathrm{http}: / /$ lanochedelloro.com \\
\hline
\end{tabular}

Todos estos datos se incorporaron en una geodatabase gestionada a través del Sistema de Información Geográfico ArcGIS v. 10.0, georreferenciándose en sistema de coordenadas UTM, aunque tuvieron que ser re-proyectados del sistema de coordenadas geográficas European Datum 1950, al WGS 84, equivalente al ETRS 89, actual sistema geodésico de referencia oficial en España (R.D. 1071/2007, de 27 de julio).

A continuación, y con el fin de poder precisar todavía más la ubicación tanto de los nidos como de los sectores de escalada, se recurrió al manejo de la Ortofoto del Plan Nacional de Ortofotografía Aérea, PNOA 2009, con resolución de 0,5 metros, apoyado en la información topográfica del Mapa Topográfico Nacional escala 1:25.000. Esta base permitió la georreferención de la información obtenida a partir de la bibliografía y de los diversos blogs (Tabla 1) especializados en deporte de escalada.

Por otra parte, los datos de nidificación de Quebrantahuesos procedentes de la base de datos sobre la población reproductora de quebrantahuesos en Aragón (FCQ-GA), han permitido extraer las coordenadas de ubicación de los nidos dentro del área del Plan de Recuperación del Quebrantahuesos en Aragón, en su sector pirenaico. Estos datos provienen de un sistemático seguimiento en el tiempo de la especie (desde 1995), por lo que era necesario también un análisis y valoración del nivel de precisión de los mismos.

Al mismo tiempo, hubo que proceder a la corrección de ubicaciones de los nidos, por una parte derivada de los datos ubicados mediante topografía 1:50.000 y 1:25.000 en papel durante los primeros años en los que no se contó con dispositivos GPS en campo y por otra aquellos que asumían errores derivados de la localización con GPS de nidos con anterioridad al año 2000, y que incorporaban errores de precisión aleatorios introducidos por el Departamento de Defensa de los EE.UU. debido al carácter militar del sistema GPS. 
Para ello, se diseñó una metodología destinada a la corrección mediante la utilización del Modelo Digital de Elevaciones de Aragón y la cartografía derivada del mismo, como el mapa de pendientes y su posterior reclasificación, completándose esta información con el empleo del SIOSE (Sistema de Información de Ocupación del Suelo en España) para incorporar información relativa a los usos del suelo, permitiendo filtrar y ubicar con mayor precisión los datos.

Tras la digitalización de todos los sectores de escalada conocidos para la provincia de Huesca, se crearon áreas de influencia o "Buffers" para cada nido conocido, tomando como referencia el punto de ubicación de éste. Estos "Buffers" se diseñaron en anillos concéntricos de 100, 300 y 500 m., según los datos de distancia de afección de las actividades antrópicas respecto a los nidos propuesta por otros autores (Arroyo y Razin, 2006, Longares, 2011) y datos propios (Figura 3).

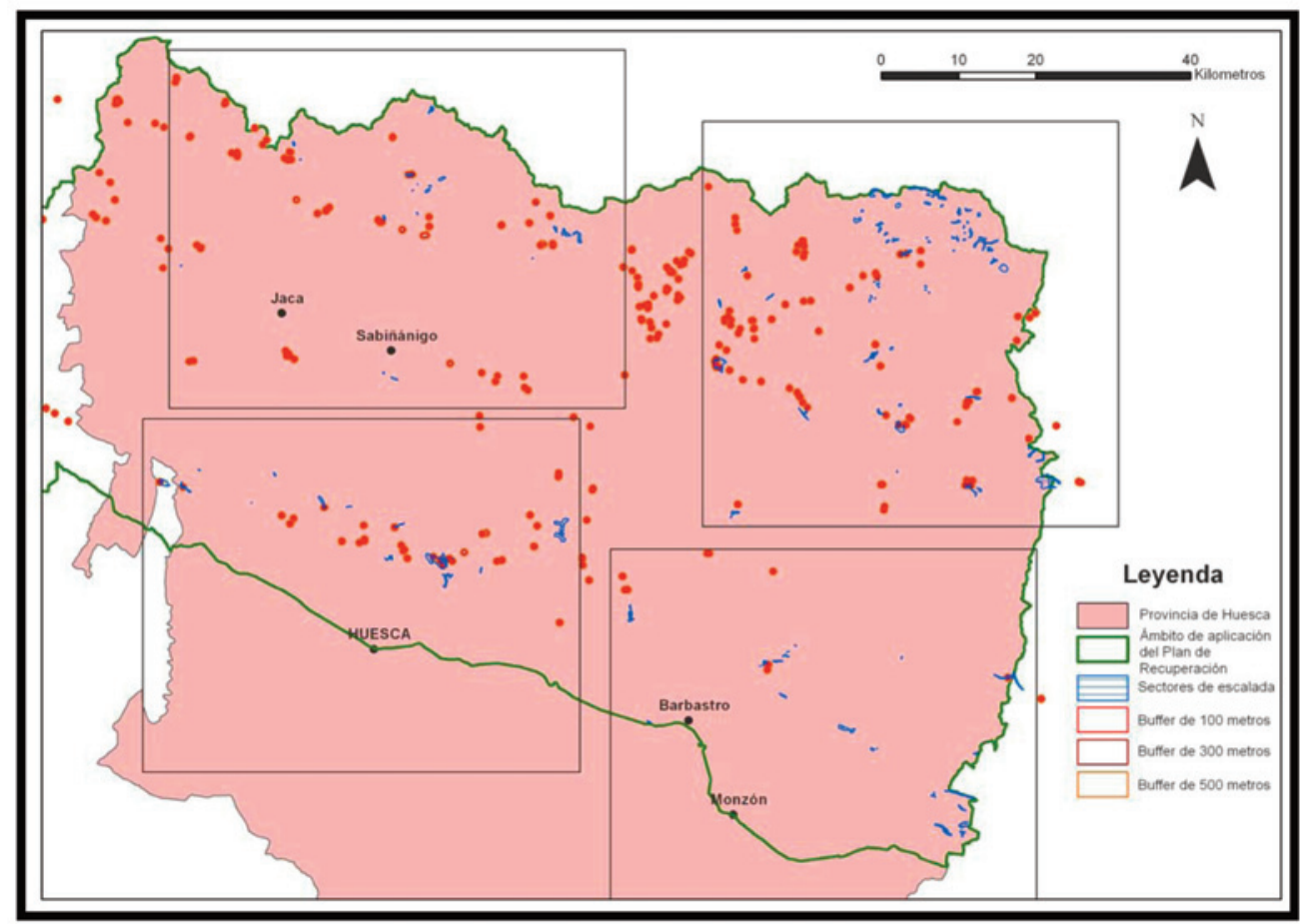

Figura 3. Sectores de escalada, puntos de nidificación de Quebrantahuesos en Aragón y "Buffers" correspondientes. 
El proceso de mejora de ubicación de los nidos de la UR, se basó en un método automatizado que descartaba las zonas cuya pendiente fuera inferior al 30\%, de tal manera que se eliminaron aquellos espacios que topográficamente no se correspondían con paredes o cortados y donde, por lo tanto, la especie no puede seleccionar espacios para su reproducción. Así se elaboró una cartografía de pendientes con la herramienta "Slope" de ArcGis a partir del Modelo Digital de Elevaciones (MDE), estableciéndose dos únicas categorías: 0 (aquellos píxeles cuya pendiente es inferior al 30\%) y 1 (aquellos píxeles cuya pendiente es superior al 30\%). Con el fin de filtrar estos datos únicamente topográficos, se procedió a cruzar la información con la cartografía de usos del suelo SIOSE, pudiendo eliminar de esta forma, aquellas zonas que no se correspondían con zonas de roquedo, asignando también varias categorías:

A. Píxeles cuyo uso principal del suelo sea "suelo desnudo"(Categoría SIOSE número 333) y como uso secundario "terrenos sin vegetación" (Categoría SIOSE número 330). También se elegirán los que posean como uso principal "afloramientos rocosos y roquedos" (Categoría SIOSE número 352) y como uso secundario "roquedos" (Categoría SIOSE número 350).

B. Aquellos píxeles cuyo uso del suelo no se corresponda con los anteriores.

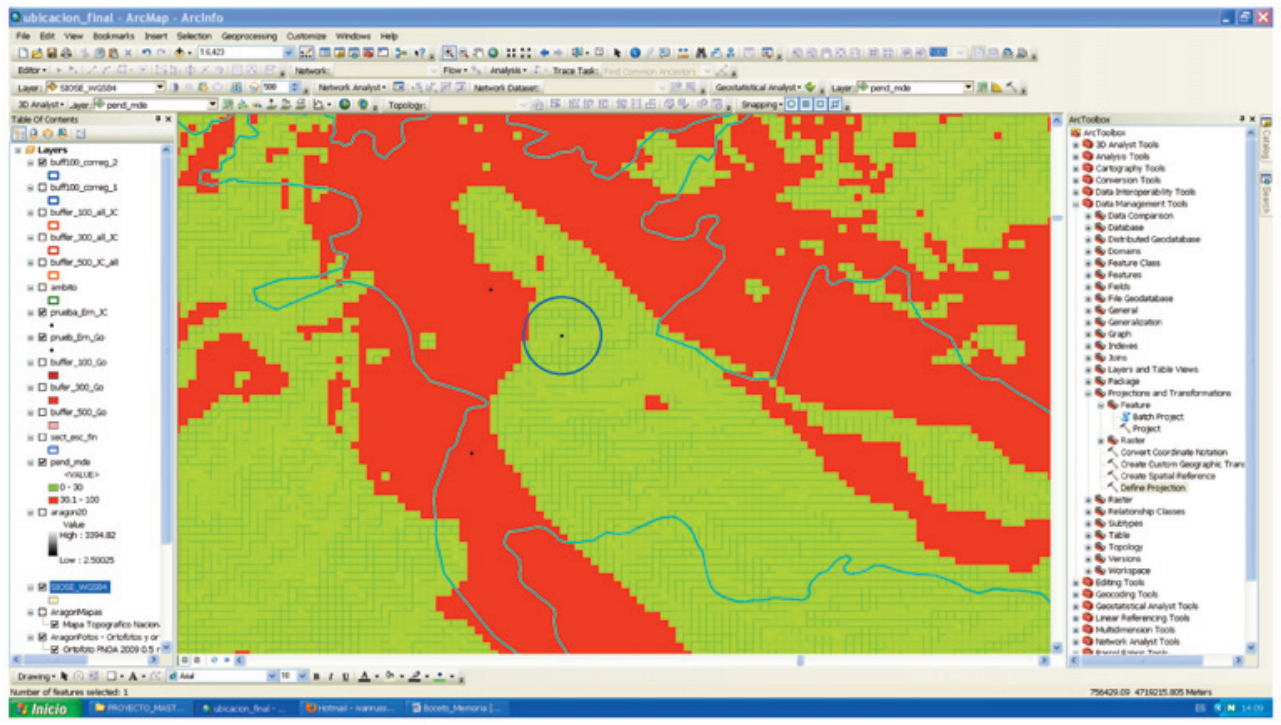

Figura 4. Ejemplo en pantalla de ArcGIS de ubicación incorrecta de punto de nidificación y utilización de la información de usos de suelo y pendiente para su corrección automática. Los píxeles (100 x 100 metros) rojos se corresponden con las zonas de pendiente favorable a la ubicación de nidos, mientras que los amarillos son zonas desfavorables, los círculos azules son las áreas de influencia delimitadas para las ubicaciones de los nidos (100 metros de radio), por su parte los usos de suelos aparecen delimitados por polígonos sin relleno con líneas azules. 


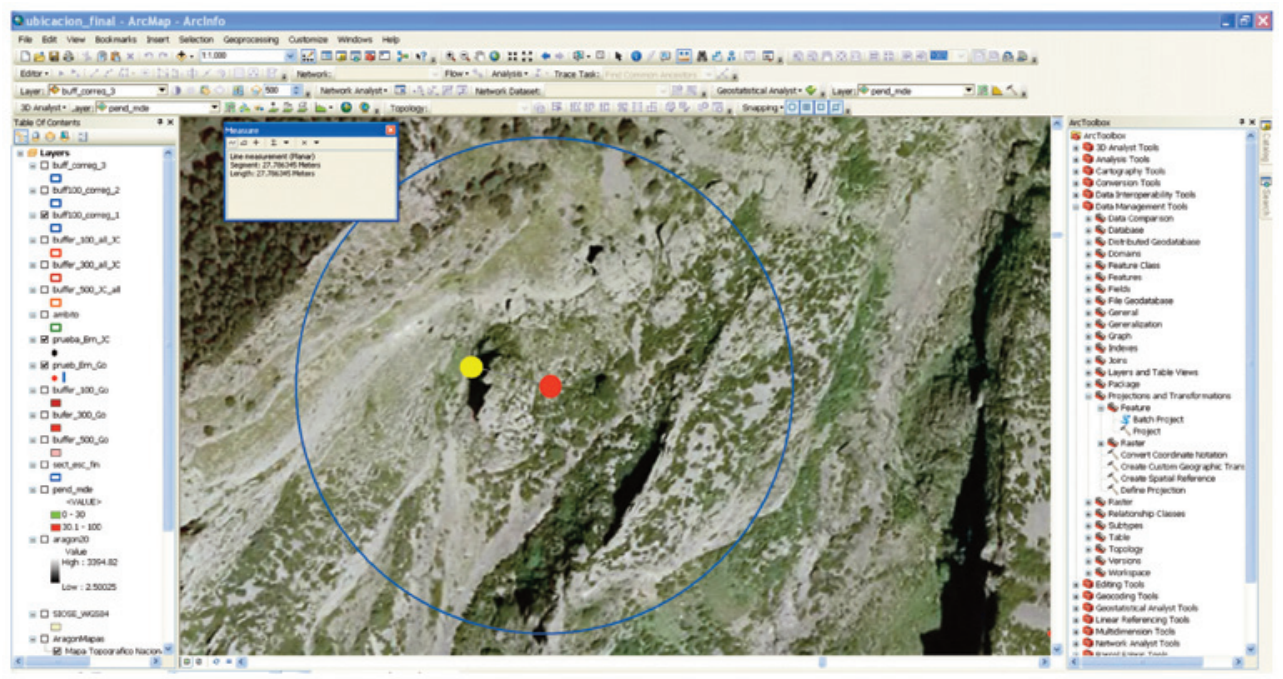

Figura 5. Ejemplo de ubicación incorrecta (rojo) de punto de nidificación y utilización de ortofografía del PNOA 2009 para ubicación (amarillo) corregida de forma manual.

Fuente: Elaboración propia.

Establecidas y preparadas las variables, se crearon de nuevo "Buffers" de radio 100 metros alrededor del punto de ubicación de los nidos. Dentro de esa zona de 100 metros se discriminaron todos los píxeles que cumplían las condiciones anteriormente descritas de los que no las cumplen (Figura 4), siendo revisados posteriormente con la ortofotografía del PNOA como base, para proceder a corregir manualmente (Figura 5) aquellos puntos que todavía no fueran lo suficientemente precisos en su ubicación.

Posteriormente y sobre los puntos de nidificación y sectores de escalada correctamente georreferenciados, se realizó una búsqueda por atributos y otra por localización para identificar todos aquellos nidos que incluían en el radio de influencia de sus "Buffers" algún sector de escalada.

Con este análisis se pudo determinar la influencia de los sectores de escalada sobre los nidos por la distancia relativa entre ambos y la concentración del número total de vías de escalada en cada uno de los diferentes sectores, datos que permitieron establecer una jerarquización de la probabilidad de la afección a la nidificación del quebrantahuesos en función de la variable distancia y concentración (Tabla 2). 
Tabla 2. Valores establecidos de la posible afección, según en número de vías de escalada presentes en los sectores analizados en relación con los puntos de nidificación del Quebrantahuesos

\begin{tabular}{|l|c|}
\hline Afección & $N^{o}$ de vías \\
\hline Baja & 25 \\
Media & $25-50$ \\
Alta & $50-100$ \\
Extrema & $>100$ \\
\hline
\end{tabular}

Fuente: Elaboración propia.

\section{Resultados}

Los resultados de este trabajo han permitido elaborar una memoria detallada del nivel de posible afección por actividad de escalada sobre la reproducción del Quebrantahuesos en Aragón, que han quedado plasmados de forma resumida en una fichas detalladas de cada una de las UR y que han sido facilitadas a los organismos encargados de llevar a cabo el Plan de Recuperación del Quebrantahuesos en la C.A. (Figura 6).

Por otra parte, desde el punto de vista de la biología de la especie, se constata que de un total de 315 nidos conocidos de Quebrantahuesos en Aragón (datos de 2011), el $11,75 \%$ de estos $(n=37)$ presentan una valoración alta en cuanto al riesgo de afección por la actividad de escalada en roca que se desarrolla en su entorno próximo. De estos, el 76\% ( $n=28)$ se sitúan en valores altos y extremos (Figura 7) por la proximidad y grado de concentración de vías de escalada en su entorno.

\section{Conclusiones}

El presente estudio se sitúa dentro de los métodos de trabajo en relación con el análisis geoespacial relacionado con la conservación de especies. Los trabajos hasta el momento existentes en esta línea de investigación aplicada no han desarrollado en ningún momento técnicas de análisis apoyadas en la nuevas tecnologías de la información geográfica como los SIG u otros que lleven a cabo un desarrollo cartográfico y estadístico paralelo. En este sentido, los únicos trabajos a nivel nacional que abordan esta temática (Ariño, 2009 y Arteaga, 2011) tan sólo lo tratan desde el punto de vista de la gestión y la divulgación, sin profundizar en el análisis y por supuesto sin utilizar nuevas tecnologías que aporten más precisión y mayor capacidad de análisis. 


\begin{tabular}{|c|c|c|c|c|c|c|}
\hline \multicolumn{7}{|c|}{ UNIDAD REPRODUCTORA: EJEMPLO } \\
\hline & $\begin{array}{c}\text { COORDENADAS } \\
\text { UTM }\end{array}$ & $\begin{array}{c}\text { AFECCIÓN } \\
\text { A 100 M. }\end{array}$ & $\begin{array}{c}\text { AFECCIÓN } \\
\text { A 300 M. }\end{array}$ & $\begin{array}{c}\text { AFECCIÓN } \\
\text { A 500 M. }\end{array}$ & $\begin{array}{c}\text { SECTOR DE } \\
\text { ESCALADA } \\
\text { QUE LE } \\
\text { AFECTA }\end{array}$ & $\begin{array}{c}\text { NIVEL } \\
\text { DE LA } \\
\text { AFECCIÓN }\end{array}$ \\
\hline Nido 1 & & $\mathrm{Si}$ & $\mathrm{Si}$ & $\mathrm{Si}$ & Ejemplo & $\begin{array}{c}\text { Extrema } \\
\text { (400 Vías) }\end{array}$ \\
\hline
\end{tabular}
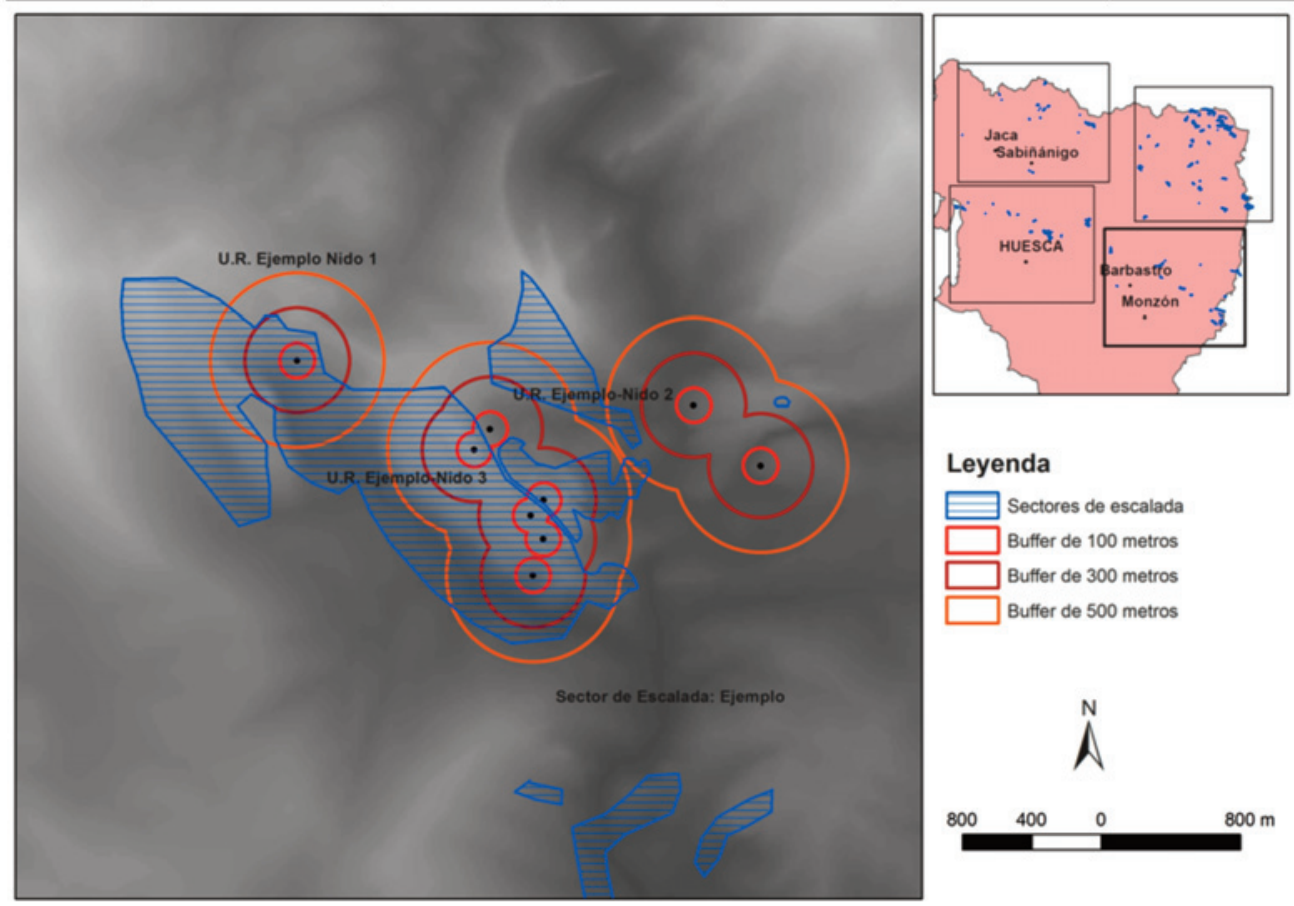

\section{Leyenda}

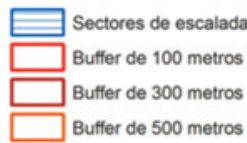

Figura 6. Ficha de valoración de la posible afección por escalada en UR. Por motivos de confidencialidad respecto a la ubicación de los nidos se ha denominado la UR y los sectores de escalada como "Ejemplo", omitiendo la base topográfica de referencia que acompaña a cada cartografía elaborada, e incorporando como base de fondo un modelo digital de terreno de resolución 20 m (Fuente: SITAR-Sistema de Información Territorial de Aragón)

Fuente: Elaboración propia.

Por tanto, consideramos que la metodología desarrollada y aplicada para este estudio concreto ha permitido profundizar en la mejora de las variables analizadas, pudiendo obtener resultados que sienten las bases para la gestión por parte de la administración o un posterior estudio más detallado. 


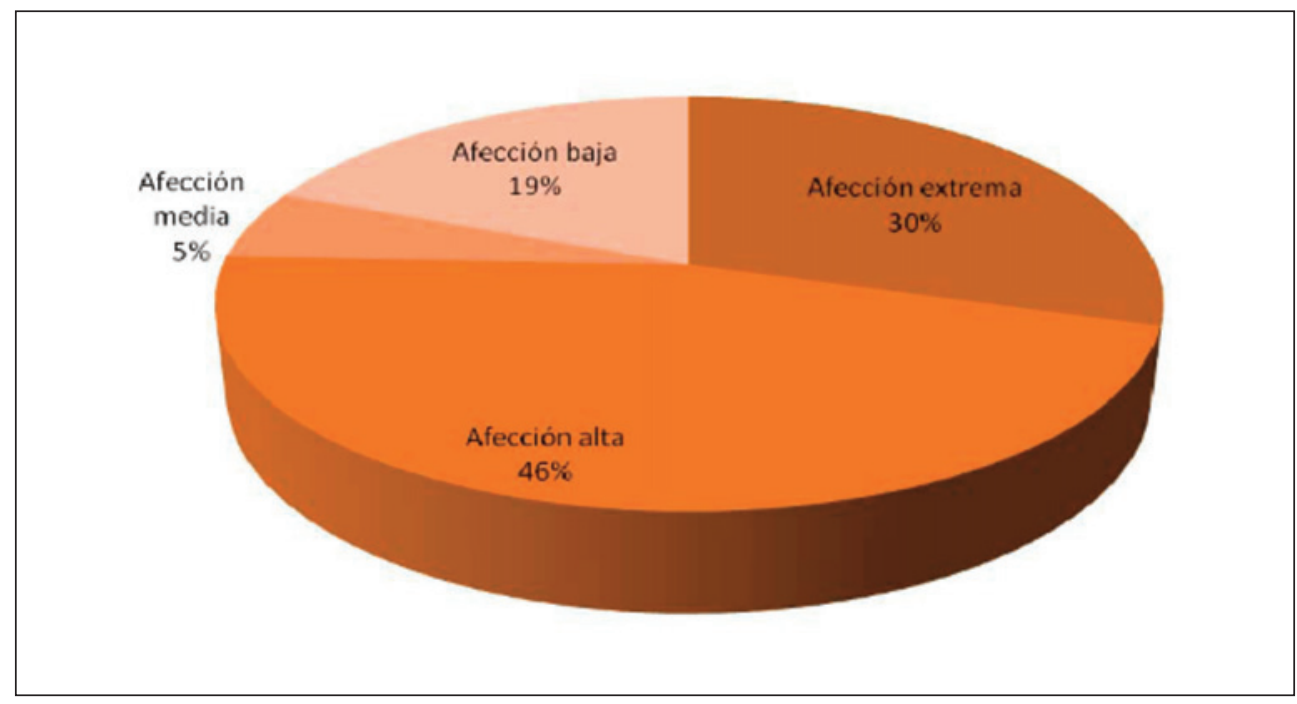

Figura 7. Distribución porcentual de los niveles de probable afección de los nidos de Quebrantahuesos $(n=37)$ influidos por la escalada deportiva en Aragón.

Fuente: Elaboración propia.

El estudio, ha puesto también de manifiesto la idoneidad de poder realizar un análisis sobre cartografía de mayor detalle usando como base el MDT con paso de malla de $5 \mathrm{~m}$. denominado sistema LIDAR, sobre los que referenciar tanto los sectores de escalada como los lugares de nidificación, (no disponible en el momento de la realización de este trabajo), pero que con toda seguridad en un futuro próximo permitirá afinar en los procesos de georreferenciación.

Por otra parte la utilización de la base de datos de usos del suelo del SIOSE, aunque correcta en lo relativo a usos del suelo por parte del hombre, en el caso de nuestro trabajo ha mostrado no discriminar suficientemente ambientes relacionados con paredes escarpadas (hábitat seleccionado para la reproducción por el Quebrantahuesos), del roquedo y del suelo desnudo en general, aspecto que hace necesario en algunos casos una labor detallada de fotointerpretación para solventar esta deficiencia y dar mayor rigurosidad al análisis.

Hay que destacar que los resultados obtenidos exponen una problemática que podría estar afectando al éxito reproductor de la especie en UR concretas y que ya ha sido presentada por algunos autores como una causa del fracaso reproductor de forma directa (Gil, 2011) o indirecta como molestias humanas (Richardson y Miller 1997; Arroyo y Razin, 2006). Por lo tanto los resultados dotan de una información básica para la 
gestión del territorio ocupado por la especie e incluido en el Plan de Recuperación del Quebrantahuesos en Aragón, por lo que la actividad de escalada debería ser regulada según el Decreto 45/2003 que dicta: "Evaluar los riesgos derivados las actividades deportivas y recreativas no reguladas, y, en su caso, restringirlas de manera coherente con la conservación de la especie y con el mantenimiento de la funcionalidad de las Áreas Críticas", situación esta que no se lleva a cabo en la actualidad.

En este sentido, el presente trabajo ya ha llevado a cabo la primera fase de lo dictado por el Decreto 45/2003, la evaluación y el análisis detallado, estableciendo una zonificación y señalando aquellas UR que pueden presentar mayores problemas en relación con la actividad deportiva, por lo que los resultados de este trabajo adquieren mayor importancia al dotar a la administración de un documento de partida que le permite iniciar con certeza los procesos de regulación necesarios, concentrando al mismo tiempo sus esfuerzos en aquellos sectores de escalada más problemáticos.

\section{Agradecimientos}

Queremos expresar nuestro agradecimiento a todas las personas que han colaborado en el desarrollo de estos trabajos y especialmente al Gobierno de Aragón, Departamento de Geografía y Ordenación del Territorio (Universidad de Zaragoza), profesores del Máster Tecnología de la Información Geográfica y al personal de la FCQ.

\section{Bibliografía}

Antor, R.; Margalida, A. y Heredia, R., 2004. Quebrantahuesos (Gypaetus barbatus). En A. Madroño, A., C. González y J.C. Atienza (Eds.). Libro Rojo de las Aves de España: 125-129. Dirección General para la Biodiversidad-SEO/BirdLife. Madrid.

Arteaga, Z., 2011. Escalada en roca y medio ambiente en Bizkaia. Edita: Diputación Foral de Bizkaia.

Ariño, X., 2009. La gestión de la escalada en los Espacios Naturales Protegidos. El caso del Parc Natural de la Muntanya de Montserrat. Universitat Autònoma de Barcelona-Federació d'Entitats Excursionistes de Catalunya (FEEC).

Arroyo, B. y Razin, M., 2006. Effect of human activities on bearded vulture behaviour and breeding success in French Pyrenees. Biol. Conserv. 128, 276-284.

BOA nํㅡㄹ 29 de 12 de Marzo de 2003. Plan de Recuperación del Quebrantahuesos en Aragón.

Del Hoyo, J.; Elliott, A. y Sargatal, J., 1994. Handbook of the Birds of the World. Vol. 2: 125. New World Vultures to Guineafowl. Lynx Edicions.

Gil, J.A., 2009. Evaluación de riesgos de colisión y electrocución de los tendidos eléctricos de las ZEPAs del ámbito de aplicación del Plan de Recuperación del Quebrantahuesos (Gypaetus barbatus) en Aragón. Pirineos 164: 165-172. Jaca. 
Gil, J.A., 2011. Impacto de las actividades humanas en las zonas de cría del Quebrantahuesos (Gypaetus barbatus) en los Pirineos. Naturaleza Aragonesa no 27: 22-27. Sociedad de Amigos del Museo Paleontológico de la Universidad de Zaragoza.

Gil, J.A. y De Frutos, A., 2008. Revisión de los envenenamientos de quebrantahuesos (Gypaetus barbatus) en los Pirineos (España) 1994-2007. En: Actas del seminario mortalidad por intoxicación en aves necrófagas. Problemática y soluciones: 49-65. Aínsa, Huesca. Departamento de Medio Ambiente del Gobierno de Aragón y Fundación para la Conservación del Quebrantahuesos.

Gil, J.A., Díez, O., Lorente, L., Báguena, G., Chéliz, G. y Ascaso, J.C., 2009. On the trail of the Bearded Vulture (Gypaetus barbatus): world distribution and population. Fundación para la Conservación del Quebrantahuesos (FCQ).

Guinda, F. y Logroño, C., 2012. "Dónde escalar en España”. Ediciones Desnivel, 266 pp.

Hiraldo, F., Delibes, M. y Calderón, J., 1979. El Quebrantabuesos (Gypaetus barbatus). (L.). Sistemática, taxonomía, biología, distribución y protección. Monografías 22: 114-121. Ministerio de Agricultura, ICONA, Madrid, España.

Longares, L.A., 2011. Zoogeografía ecológica del Quebrantabuesos (Gypaetus barbatus) en Aragón. Análisis y valoración de los factores ecogeográficos en relación con la reproducción. Universidad de Zaragoza. ISBN: 978-84-691-7186-8. <http://zaguan.unizar.es/record/1893>

Margalida, A., Heredia, R., Razin, M. y Hernández, M., 2008. Sources of variatios in mortality of the Bearded Vulture Gypaetus barbatus in Europe. Bird Conservation International 18: 1-10.

Ostermann, O.P., 1998. The need for management of nature conservation sites designated under Natura 2000. Journal of Applied Ecology 35: 968-973.

Richardson, C.T. y Miller, C.K., 1997. Recommendations for protecting raptors from human disturbance: a review. Wildlife Society Bulletin 25, 634-638.

Sánchez-Castilla, E., Gil, J.A., Longares, L.A. y Alcántara, M., 2008. Hábitat potencial para el quebrantahuesos en áreas de antigua distribución del Sistema Ibérico oriental (Aragón). Xiloca, 36: 85-96. 
